\title{
CONSIDERATIONS OF NDT QUALITY IN FRACTURE-CRITICAL INSPECTIONS FOR STEEL BRIDGES
}

\author{
H.-Y. Chung ${ }^{1}$, L. Manuel' ${ }^{2}$ and K. H. Frank ${ }^{3}$ \\ ${ }^{1}$ Department of Civil Engineering, National Cheng Kung University, Tainan 701, Taiwan, ROC \\ ${ }^{2}$ Department of Civil Engineering, University of Texas, Austin, TX 78712, USA \\ ${ }^{3}$ Department of Civil Engineering, University of Texas, Austin, TX 78712, USA
}

\begin{abstract}
Non-destructive testing (NDI) techniques are routinely used in bridge inspection. However, detection accuracy, accessibility, cost and consequences of false indication are all factors to consider when selecting the NDI techniques to be used. A probabilistic method is proposed to help select the most suitable NDI technique and an associated optimal schedule for fracture-critical member/detail inspections on a specified steel bridge. The Probability of Detection (POD) curve for each NDI technique which denotes its detection accuracy is employed. In addition, by combining probability calculations based on the use of the POD curve together with numerical Monte Carlo simulations for crack propagation in the fracture-critical detail, a total cost function is formulated that includes the expected cost of inspections, repair and failure resulting from the chosen NDI method and alternative inspection schedules. In summary, the selection of an NDI method with an associated inspection schedule for fracture-critical inspections is formulated as an optimization problem which utilizes appropriate constraints on inspection intervals and on a minimum acceptable (target) structural reliability. One case study for a box girder bridge is presented to demonstrate the proposed probabilistic method for selecting the optimal NDI method and corresponding inspection schedule.
\end{abstract}

\section{KEYWORDS}

POD, NDI, steel bridge, fatigue, fracture-critical member, Monte Carlo simulation

\section{INTRODUCTION}

Inspection accuracy, accessibility, frequency, cost, and consequences of detection failures (misses) or false indications (false calls) must be considered when selecting the appropriate non-destructive inspection (NDI) technique for steel bridges. Though other reliability-based fatigue inspection scheduling methods in the offshore industry, such as the method proposed by Sorensen et al. (1991) include inspection quality in their analyses, it is difficult to calibrate the inspection quality coefficients and other parameters in their models so as to propose use of any single NDI technique (over alternatives). Also, these methods generally demand complicated reliability algorithms to obtain optimal inspection results; this limits their applicability to bridge maintenance with associated tighter budgetary constraints. An intuitive probabilistic approach for dealing with inspection quality is proposed in this paper to select the most economical NDI method. On completion of each analysis that involves Monte Carlo simulations, the method recommends a single NDI technique and accompanied inspection schedule for fracture-critical members in a steel bridge that guarantees a specified acceptable safety level through the planned service life of the bridge. The actual Probability of Detection (POD) functions associated with various NDI techniques are employed as the NDI detectabilities. By combining probability calculations based on the use of the POD functions together with Monte Carlo simulations of the crack growth for the fracture-critical member, a total 
cost function is formulated that includes the cost of inspections and failure that result with each alternative NDI technique and inspection schedule. The selection of an NDI technique with an associated inspection schedule is modeled as an optimization problem. The POD function corresponding to the NDI technique and the inspection interval are optimization variables for this problem. With appropriate constraints on inspection intervals and on a minimum (target) safety level, an optimal combination of NDI technique and inspection schedule that yields the minimum total cost and ensures the prescribed acceptable safety level for the specified detail can be obtained.

\section{PROBABILITY OF DETECTION}

The four possible outcomes of any NDI procedure are: true positive (hit), false negative (miss), false positive (false call) and true negative (correct accept). The probability of detection (POD) of a crack of a given size is the conditional probability of a true positive call given that a crack with that size exists. Hence, from repeated inspections, an estimate of the POD can be obtained as follows:

$$
P \hat{O} D=\frac{N_{\mathrm{TP}}}{N_{\mathrm{TP}}+N_{\mathrm{FN}}}
$$

where $P \hat{O D}=P O D$ estimate for a specific crack size; $N_{\mathrm{TP}}=$ number of true positive calls; $N_{\mathrm{FN}}=$ number of false negative calls. After introducing cracks of various sizes into test specimens and performing inspections, $P O D$ estimates for various crack sizes from different NDI techniques can be obtained. Generally, two analysis approaches - the Hit/Miss method (Berens and Hovey, 1981) and the Signal Response method (Berens, 1989)- are employed to formulate the POD function, $P O D(a)$, for any crack size, $a$, with any NDI technique.

\section{FATIGUE CRACK GROWTH MODEL}

The basic fatigue crack growth relation can be derived from the Paris' Law (1963) and Linear Elastic Fracture Mechanics (LEFM) as follows:

$$
\int_{a_{0}}^{a_{N}} \frac{d a}{[F(a) \sqrt{\pi a}]^{m}}=C \cdot N \cdot S_{R E}^{m}
$$

where $N=$ number of stress cycles; $a=$ crack size; $a_{0}=$ the initial crack size; $a_{N}=$ the crack size after $N$ stress cycles; $F(a)=$ the geometry function of the crack tip; $C=$ fatigue growth parameter; $m=$ fatigue growth exponent; $S_{R E}=$ the effective stress range. If $\Psi(a)$ is defined as the indefinite integral of the left side term of Eqn. 2, the crack growth function can then also be represented as:

$$
a_{N}=\Psi^{-1}\left[\Psi\left(a_{0}\right)+C \cdot N \cdot S_{R E}^{m}\right]
$$

The number of stress cycles, $N$, in steel bridges can be specifically modified as a function of average daily truck traffic $(A D T T)$, cycles per truck passage $\left(C_{s}\right)$ and service years $(Y)$. For structural details and components in a steel bridge, the main source of fatigue loadings comes from the vehicles, especially trucks, passing through the bridge. To account for the variable-amplitude stress ranges resulted from the random truck traffic, the effective stress range $S_{R E}$ proposed by Schilling et al. (1978), which represents the global effect of various stress ranges occurred in the detail, can be expressed as follows:

$$
S_{R E}=\left\{\sum_{i=1}^{n} \gamma_{i} \cdot S_{R, i}^{3}\right\}^{1 / 3}
$$

where $\gamma_{i}=$ ratio of the number of $S_{R, i}$ stress range amplitude cycles to the total number of cycles. Generally, the material properties $(C$ and $m)$, initial crack size $\left(a_{0}\right), A D T T$ and $C_{s}$ can be described as random variables in the crack growth function, $a_{N}$ (Eqn. 3 ) as uncertainties. Therefore, the crack growth curve is random over the service years of the detail, and can be simulated by Monte Carlo simulation technique. 


\section{PROBABILITY OF DETECTING AND NOT DETECTING A CRACK}

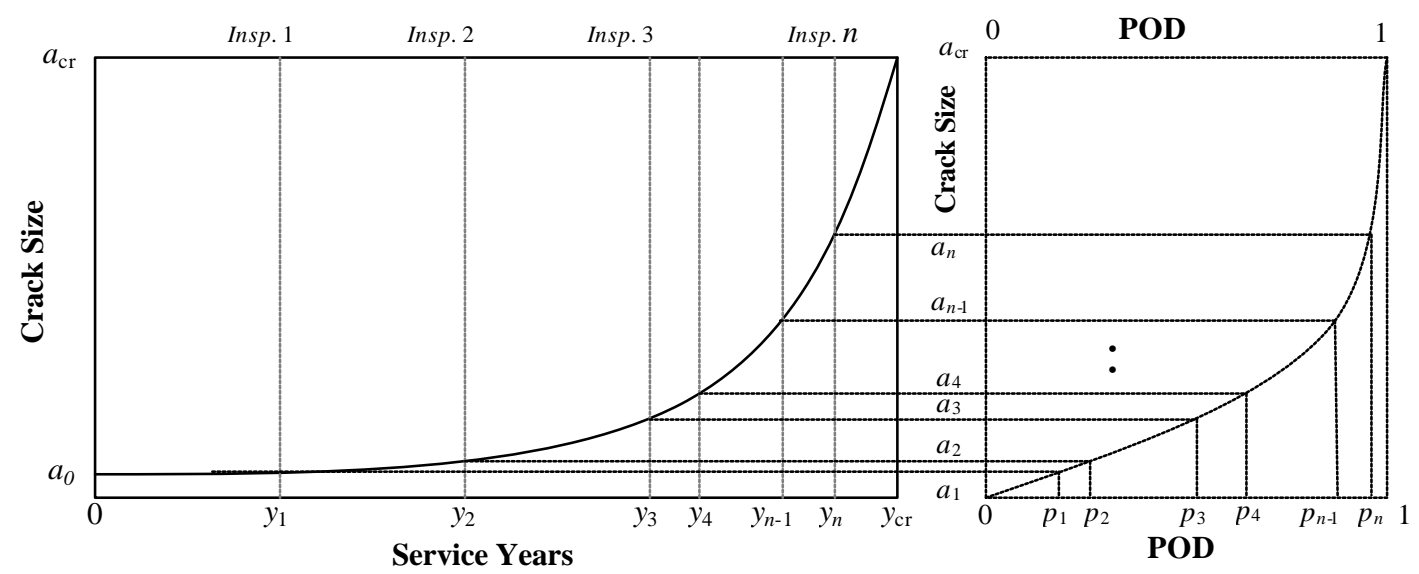

Figure 1: Probability of Detection Mapping for a Crack Growth Curve

Suppose a crack growth curve (from Eqn. 3) for a fracture critical bridge detail is obtained, and $n$ non-destructive fatigue inspections are performed constantly on this detail. After reflecting (mapping) the crack size $a_{i}$ at the $i^{\text {th }}$ inspection on the POD curve diagram related to the applied NDI technique, the probability of detecting a given crack size $a_{i}$, denoted as $p_{i}$, can be determined (see Fig. 1). Therefore, the probability of not detecting a crack, $P_{n d}$, and the probability of detecting a crack, $P_{d}$, during the detail life can be expressed as:

$$
\begin{gathered}
P_{n d}=\prod_{i=1}^{n}\left(1-p_{i}\right) \\
P_{d}=1-P_{n d}=1-\prod_{i=1}^{n}\left(1-p_{i}\right)
\end{gathered}
$$

Considering the uncertainties in the crack growth model described in the previous section, the Monte Carlo technique can be applied to simulate possible crack growth curves. Each crack growth simulation $j$ provides two values of interest, one is the probability of not detecting a crack $P_{n d, j}$ and the other value is the number of inspections $n_{j}$. Accumulating the results of all crack growth simulations, the expected values of Eqn. 5 and Eqn. 6 can be obtained as follows:

$$
\begin{gathered}
E\left(P_{n d}\right)=\frac{1}{N_{\text {sim }}} \sum_{i=1}^{N} P_{n d, i} \\
E(n)=\frac{1}{N_{\text {sim }}} \sum_{i=1}^{N} n_{i}
\end{gathered}
$$

where $N_{\text {sim }}=$ number of simulations. With sufficient simulations, the converged results of $E\left(P_{n d}\right)$ and $E(n)$ can be achieved. For the given POD curve of an NDI technique and the accompanied inspection schedule, $E\left(P_{n d}\right)$ represents the risk that the applied NDI technique will fail to detect the existing crack in a detail through the whole fatigue life of the detail. $E(n)$ corresponds to the most likely number of inspections for which the selected NDI method might be performed during the fatigue life of the detail in the future. It should be noted that the crack growth simulations here do not involve any repair activity.

\section{OPTIMAL NDI TECHNIQUE}

To transform the selection of a proper NDI technique to an optimization problem, a cost function reflecting the consequence of choosing a NDI technique and the associated inspection schedule for the considered detail and two constraints related to structural safety and schedule feasibility need to be created. With the help of $E(n)$ and $E\left(P_{n d}\right)$ obtained from Monte Carlo simulations, the optimization problem for selecting an optimal NDI technique and associated inspection schedule may be stated as follows: 


$$
\min _{\mathrm{POD}, y_{\mathrm{int}}} C_{T}=K_{I} E(n)+K_{F} E\left(P_{n d}\right)
$$

Optimization variables: POD function and inspection interval, $y_{\text {int }}$

Subjected to: $E\left(P_{n d}\right)<P_{n d \text {, max }} ; y_{\text {min }}<y_{\text {int }}<y_{\text {max }}$

where $C_{T}=$ cost function; $K_{I}=$ cost of a single inspection of the specified detail; $K_{F}=$ cost associated with a failure. $P_{n d \text { max }}=$ the maximum allowable probability of not detecting a crack (i.e., the minimum acceptable safety level); $y_{\min }$ or $y_{\max }=$ the lower or upper bound of inspection interval. In cost function $C_{T}$, the first term $\left(K_{I} E(n)\right)$ represents the expected cost of inspections over the service life. Note that, if an NDI technique continuously fails to detect the growing crack in the detail, the expected fatigue failure probability will be high. Hence, $E\left(P_{n d}\right)$ gives an indication of the likelihood of fatigue failure for the detail over the service life as a result of failure to detect a growing by the selected NDI technique. The second term $\left(K_{F} E\left(P_{n d}\right)\right)$ in $C_{T}$, therefore, denotes the possible costs relating to the failure consequences of the considered detail. $P_{n d \text { max }}$, defined as the maximum allowable probability of not detecting a crack (i.e., the minimum acceptable safety level) for the NDI technique applied on the specified fatigue detail, is applied as the first constraint to exclude combinations of NDI techniques and inspection schedules that might be deemed unsafe because $E\left(P_{n d}\right)$ is too high. In addition, the second constraint sets restrictions on the time between inspections so that this inter-inspection interval is neither too large nor too short. Upon minimizing the total cost, an optimum inspection interval, $y_{\text {int }}$, may be found. In addition, by changing the POD functions, i.e., changing the NDI technique, the total cost corresponding to the different POD functions may be compared so as to finally yield the optimization solution for the NDI technique and an associated inspection schedule.

\section{NUMERICAL EXAMPLE}

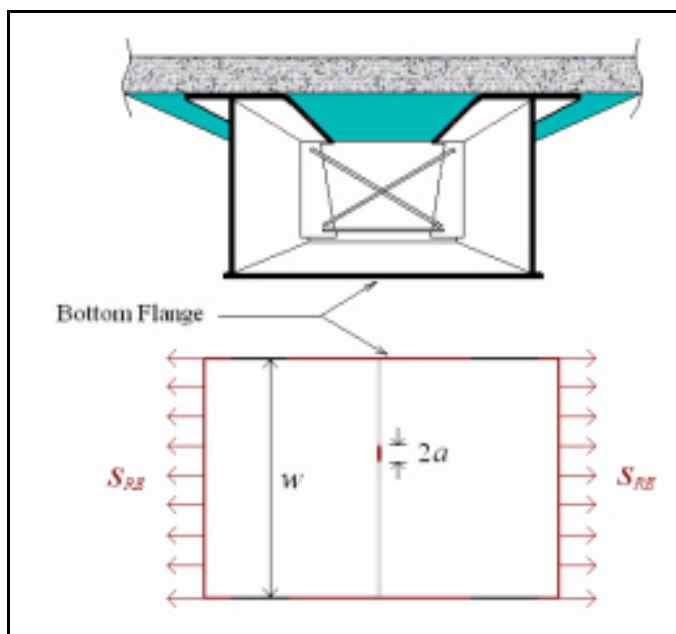

(a) Fracture Critical Detail in Box Girder Bridge

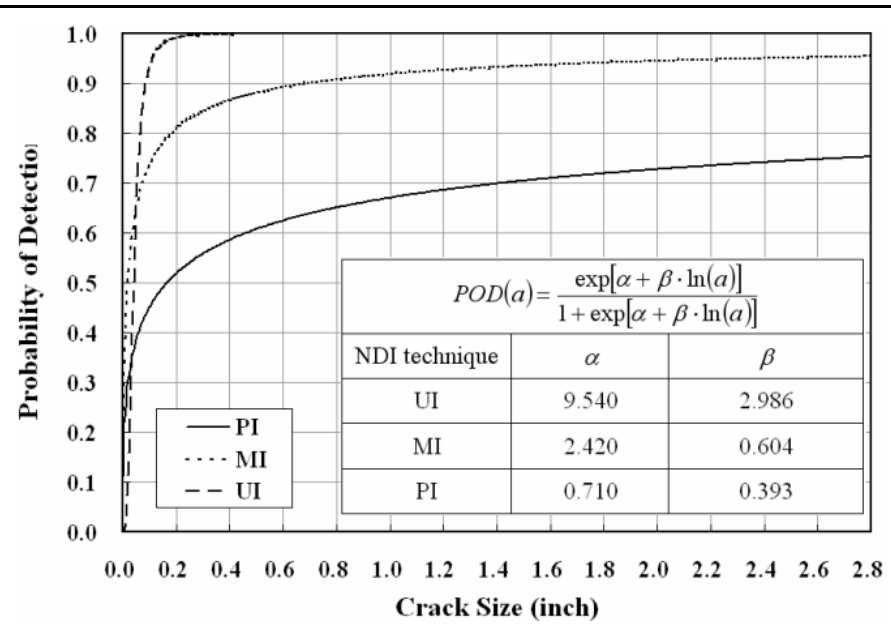

(b) POD Curves for UI, MI and PI (Berens and Hovey, 1981)

Figure 2: Illustration of the Considered FCM and POD Curves of the Three Selected NDI's

TABLE 1

RELATED RANDOM VARIABLES FOR CENTER CRACK IN BOTTOM FLANGE

\begin{tabular}{|l|c|c|c|}
\hline \multicolumn{1}{|c|}{ Variable } & Type & Mean & COV \\
\hline$a_{0}$ (initial crack size, in.) & lognormal & 0.020 & 0.500 \\
\hline$a_{\mathrm{c}}$ (critical crack size, in.) & constant & 2.000 & 0.000 \\
\hline$a_{R}$ (crack size for repair, in.) & constant & 0.200 & 0.000 \\
\hline$C$ (fatigue growth parameter) & lognormal & $2.05 \times 10^{-10}$ & 0.630 \\
\hline$m$ (fatigue growth exponent) & normal & 3.000 & 0.100 \\
\hline
\end{tabular}


Two full-penetration butt welds in the bottom (tension) flange of a newly built steel box girder bridge are studied in this example for which we seek an optimal NDI technique and an optimal inspection schedule for future service years. It is assumed that failure of the butt weld detail will result in collapse of the box-girder span. An inherent flaw is assumed to exist in the butt welds of the 60 in. width $(w)$ bottom flange as shown in Fig. 2a. Random variables related to the crack and its growth are listed in Table 1, which are adapted from one described by Zhao et al. (1994). The average daily truck traffic, $A D T T$, and the number of stress cycles per truck passage, $C_{S}$, for the box girder bridge are taken to be 600 and 1.0, respectively. A Rayleigh distribution is employed to model the stress range spectrum for the bottom flange of the bridge and $S_{R 0}$ is taken as $6.334 \mathrm{ksi}$ for the stress range. Three NDI techniques - ultrasonic inspection (UI), magnetic particle inspection (MI) and penetrant inspection (PI) - are considered here for the butt weld detail. The POD functions for these three techniques, shown in Fig. 2b, are based on POD data from the flat plate testing results collected by Rummel and Matzkanin (1997). The relative costs of the three types of nondestructive inspections and of the cost of failure are considered here: $K_{I, P I}: K_{I, M I}: K_{I, U I}: K_{F}=1.0: 1.2: 1.5$ : $2.0 \times 10^{4}$. The maximum acceptable probability of not detecting a crack over the service life is taken to be 0.005 , i.e. $E\left(P_{n d}\right)<0.005$ or $P_{n d, \max }=0.005$, in this example.

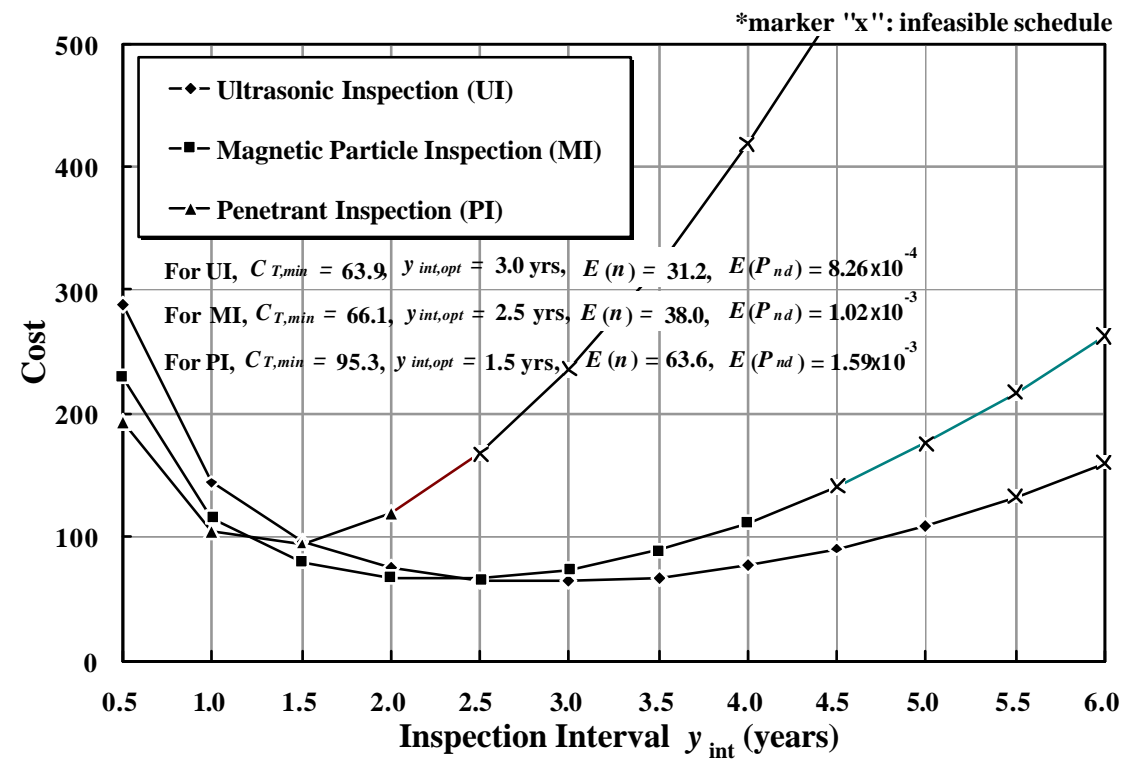

Figure 3: Cost Comparison of UI, MI and PI in Various Fixed-Interval Schedules

Fig. 3 shows total costs for the feasible and infeasible schedules with the three NDI techniques from the proposed optimization procedure. Note that each marker " $X$ " in Fig. 3 indicates an infeasible schedule that fails to meet the constraint, $E\left(P_{n d}\right)<0.005$. It can be seen that the optimal schedule is to perform ultrasonic inspections every 3 years. As shown in Fig. 3, even though a single ultrasonic inspection is more expensive than a single magnetic particle or penetrant inspection $\left(K_{I, P T}: K_{I, M T}\right.$ : $\left.K_{I, U T}: K_{F}=1.0: 1.2: 1.5\right)$, the less frequent inspections and the higher flaw detectability with the UI technique together yield lower total costs than that resulting from use of the other two NDI techniques. Both, the magnetic particle and the penetrant inspection, have lower single inspection costs, but the greater number of inspections and the higher probability of failing to detect a crack resulting from a lower flaw detectability cause higher total costs than with the ultrasonic inspections.

\section{DISCUSSIONS AND CONCLUSIONS}

A probabilistic approach for selecting an optimal NDI technique and an associated inspection schedule for fracture-critical members in steel bridges has been presented. The method builds upon LEFM fatigue analysis and the actual detection capabilities of NDI techniques for the fracture-critical member of interest, and then employs the probability of detection information in Monte Carlo simulations to formulate an optimization problem. Solution of this optimization problem yields the most suitable NDI technique and associated inspection schedule for the detail. The findings of this chapter can be summarized as follows: 
(1) In the cost- $y_{\text {int }}$ (cost vs. inspection interval, i.e. Fig. 3) diagram presented, for a given NDI technique, the total cost is controlled by the cost of inspections for schedules with short inspection intervals (i.e., the schedules involving frequent inspections). The shorter the fixed inspection interval employed in the schedule, the greater will be the cost of inspections for that schedule. For schedules with longer inspection intervals, the total cost is governed by the cost of failure. The longer the fixed inspection interval in the schedule, the greater will be the cost of failure for that schedule. Hence, a valley-shaped total cost curve is generated. After applying the $P_{n d \text {,max }}$ constraint to eliminate some infeasible schedules, the optimal schedule for an NDI technique can usually be found at the bottom of the valley-shaped total cost curve.

(2) Comparing NDI techniques, an NDI technique with a lower crack detectability will normally demand more inspections than an NDI technique with a higher crack detectability to achieve the schedule with the minimum total cost. This result is consistent with the practical experience where more inspections are needed for an NDI technique with a low crack detectability to ensure inspection quality.

(3) The optimization results are affected by the NDI detectabilities (i.e., POD functions), the constraint on the expected probability of not detecting a crack, $E\left(P_{n d}\right)$, and the relative costs of inspection and failure.

(4) Regarding the effect of crack detectability, it can be seen from the example presented (shown as in Fig. 3), that the NDI technique with the higher detectability for cracks tends to yield a lower $E\left(P_{n d}\right)$ especially for schedules with a longer inspection interval. This lower $E\left(P_{n d}\right)$ contributes in turn to lower costs of failure in the total cost. However, an NDI technique with a higher crack detectability is usually more expensive and involves higher inspection costs.

\section{REFERENCES}

Berens A.P. and Hovey P.W. (1981). Evaluation of NDE Reliability Characterization. AFWAL-TR-81-4160, Vol. 1, Air Force Wright-Aeronautical Laboratory, Wright-Patterson Air Force Base.

Berens A.P. (1989). NDE Reliability Analysis. In: Metals Handbook, Vol. 17. $9^{\text {th }}$ ed. ASM International, 689-701.

Miner M.A. (1945). Cumulative damage in fatigue, Journal of Applied Mechanics 12:3, 159-164.

Paris P. and Erdogan F. (1963). A Critical Analysis of Crack Propagation Laws. Journal of Basic Engineering, Transactions of the ASME 85:D-4, 528-534.

Rummel W.D. and Matzkanin G.A. (1997). Nondestructive Evaluation Capabilities Data Book. Nondestructive Testing Information Analysis Center, Austin TX, USA.

Schilling C.G, Klippstein K.H., Barsom J.M. and Blake GT. (1978). Fatigue of Welded Steel Bridge Members under Variable-Amplitude Loadings. National Cooperative Highway Research Program Report 188. Transportation Research Board, Washington D.C., USA.

Sorensen J.D., Faber M.H., Rackwitz R., Thoft-Christensen P. (1991). Modelling in Optimal Inspection and Repair. Proceedings of the $10^{\text {th }}$ International Conference on Offshore Mechanics and Arctic Engineering 2, American Society of Mechanical Engineers, Stavanger, Norway, 281-288.

Zhao Z, Haldar A. and Breen F.L. (1994). Fatigue-Reliability Evaluation of Steel Bridges. Journal of Structural Engineering 120:5, ASCE, 1608-1623. 